\title{
Volatility Spillovers and Causality of Carbon Emissions, Oil and Coal Spot and Futures for the EU and USA
}

\author{
C.-L. Chang ${ }^{a}$, M. McAleer ${ }^{\mathrm{b}, \mathrm{c}, \mathrm{d}, \mathrm{e}, \mathrm{f}}$ and Guangdong Zuo ${ }^{\mathrm{b}}$ \\ ${ }^{a}$ Department of Applied Economics and Department of Finance,National Chung Hsing University, Taiwan, \\ ${ }^{b}$ Department of Quantitative Finance, National Tsing Hua University, Taiwan \\ ${ }^{c}$ Discipline of Business Analytics, University of Sydney Business School, Australia \\ ${ }^{d}$ Erasmus School of Economics, Erasmus University Rotterdam, The Netherlands, \\ e Department of Quantitative Economics, Complutense University of Madrid, Spain \\ ${ }^{f}$ Institute of Advanced Sciences, Yokohama National University, Japan \\ Email: changchialin@email.nchu.edu.tw
}

\begin{abstract}
Recent research shows that efforts to limit climate change should focus on reducing emissions of carbon dioxide over other greenhouse gases or air pollutants. Many countries are paying substantial attention to carbon emissions to improve air quality and public health. The largest source of carbon emissions from human activities in some countries in Europe and elsewhere is from burning fossil fuels for electricity, heat, and transportation. The prices of fuel and carbon emissions can influence each other. Owing to the importance of carbon emissions and their connection to fossil fuels, and the possibility of Granger (1980) causality in spot and futures prices, returns and volatility of carbon emissions, crude oil and coal have recently become very important research topics. For the USA, daily spot and futures prices are available for crude oil and coal, but there are no daily futures prices for carbon emissions. For the EU, there are no daily spot prices for coal or carbon emissions, but there are daily futures prices for crude oil, coal and carbon emissions. For this reason, daily prices will be used to analyse Granger causality and volatility spillovers in spot and futures prices of carbon emissions, crude oil, and coal. As the estimators are based on QMLE under the incorrect assumption of a normal distribution, we modify the likelihood ratio (LR) test to a quasi-likelihood ratio test (QLR) to test the multivariate conditional volatility Diagonal BEKK model, which estimates and tests volatility spillovers, and has valid regularity conditions and asymptotic properties, against the alternative Full BEKK model, which also estimates volatility spillovers, but has valid regularity conditions and asymptotic properties only under the null hypothesis of zero off-diagonal elements. Dynamic hedging strategies using optimal hedge ratios are suggested to analyse market fluctuations in the spot and futures returns and volatility of carbon emissions, crude oil and coal prices.
\end{abstract}

Keywords: Carbon emissions, fossil fuels, low carbon targets and green energy, Spot and futures prices, Granger causality and volatility spillovers, Quasi likelihood ratio (QLR) test of Diagonal and Full BEKK 


\section{INTRODUCTION}

Recent research shows that efforts to limit climate change should focus on reducing emissions of carbon dioxide over other greenhouse gases or air pollutants. Many countries are paying substantially greater attention to carbon emissions to improve air quality and public health. Carbon emissions trading programs have been established at the international, regional, national, and sub-national levels.

In a scenario of 'no carbon dioxide mitigation', global temperatures would be predicted to rise by over five degrees Celsius by 2100, but cutting emissions of methane, HFCs and black carbon would reduce this rise to around one degree Celsius. The results suggest that carbon dioxide should certainly remain central to greenhouse gas emission cuts.

The largest source of carbon emissions from human activities in some countries in Europe and elsewhere is from burning fossil fuels for electricity, heat, and transportation. The price of fuel influences carbon emissions, but the price of carbon emissions can also influence the price of fuel. Owing to the importance of carbon emissions and their connection to fossil fuels, and the possibility of Granger (1980) causality in spot and futures prices, returns and volatility of carbon emissions, it is not surprising that crude oil and coal have recently become a very important public policy issue, and hence also a significant research topic.

\section{DATA}

The length of the sample period for the empirical analysis was dictated by the availability of data on carbon, coal and crude oil spot and futures prices in the EU and USA. The carbon emission trading market of the EU has the longest trading period for futures prices, but not for spot prices. The USA is the leader in developing a wide range of financial derivatives, such as futures prices, for financial, energy and commodities, but not for carbon emissions, where only spot prices are available.

Data for EU carbon emission, crude oil and coal futures are available from 1 April 2008 to 20 May 2017, and these will be analyzed in the paper. Coal spot price in the EU is available on a weekly basis. The spot prices of carbon emission and crude oil have a high correlation with the corresponding futures prices. The volume of trades in the spot market of carbon emissions is much smaller than in the futures market.

Data for crude oil are available prior to 2000. However, the data for the spot prices of coal and carbon emission start from 2006/7/17 and 2008/4/1, respectively. Therefore, the data in the empirical analysis for the European Union starts from the latest date for crude oil, coal and carbon emissions, namely 2008/4/1.

Data for carbon, coal and oil spot prices from 2016/1/5 to 2017/5/20 for the USA will also be analyzed in the paper, but data for futures prices of carbon emission are not available for the USA. Spot prices for coal and crude oil start prior to 2000. However, data for carbon emission start from 2016/1/5. Consequently, the spot price data in the empirical analysis for the USA starts from the latest date for oil, coal and carbon emissions, namely 2016/1/5.

For the USA, daily spot and futures prices are available for crude oil and coal, but there are no daily spot or futures prices for carbon emissions. For the EU, there are no daily spot prices for coal or carbon emissions, but there are daily futures prices for crude oil, coal and carbon emissions. For this reason, daily futures prices will be used to analyse Granger causality and volatility spillovers in spot and futures prices of carbon emissions, crude oil, and coal. This will be based on the Lagrange multiplier test of univariate causality in variance (strictly, causality in conditional volatility) of Hafner and Herwartz (2006), and more recently, Chang and McAleer (2017). An extension to multivariate tests of causality in conditional volatility will be a focus of the paper.

As the estimators are based on QMLE under the incorrect assumption of a normal likelihood function, we will modify the likelihood ratio (LR) test to a quasi-likelihood ratio test (QLR). A novel QLR test is given below to test the multivariate conditional volatility Diagonal BEKK model, which is used to estimate and test spillovers, and which has valid regularity conditions and asymptotic properties, against the alternative Full BEKK model, which is used to estimate spillovers, but has valid regularity conditions and asymptotic properties only under the null hypothesis of zero off-diagonal elements. Dynamic hedging strategies using optimal hedge ratios will be suggested to analyse market fluctuations in the spot and futures returns and volatility of carbon emissions, crude oil and coal prices.

QLR test statistic: 2 (quasi maximized log likelihood value under the alternative hypothesis - quasi maximized log likelihood value under the null hypothesis). 
The QLR statistic has an asymptotic chi-squared distribution under the null hypothesis, with degrees of freedom (df) equivalent to the number of off-diagonal terms in the two $\mathrm{m} \mathrm{x} \mathrm{m} \mathrm{matrices,} \mathrm{the} \mathrm{weighting} \mathrm{matrix,}$ A and the stability matrix, B, of the Full BEKK model, namely $2 \mathrm{~m}(\mathrm{~m}-1)$.

\section{METHODOLOGY}

Although financial returns are almost certainly stationary, the empirical analysis will commence with tests of unit roots based on ADF, DF-GLS and KPSS. This will be followed by an analysis and estimation of univariate GARCH and multivariate diagonal BEKK models (see Baba et al. (1985), Engle and Kroner (1995)), from which the conditional covariances will be used for testing co-volatility spillovers, that is, Granger causality in conditional volatility.

The analysis of univariate and multivariate conditional volatility models below is a summary of what has been presented in the literature, although a comprehensive discussion of the Full and Diagonal BEKK models is not available in any published source. In particular, application of the quasi likelihood ratio (QLR) test of the Diagonal BEKK model as the null hypothesis against the alternative hypothesis of a Full BEKK model does not seem to have been considered in the literature.

The first step in estimating multivariate models is to obtain the standardized residuals from the conditional mean returns shocks. For this reason, the most widely-used univariate conditional volatility model, namely GARCH, will be presented briefly, followed by the two most widely estimated multivariate conditional covariance models, namely the Diagonal and Full BEKK models.

\subsection{Univariate Conditional Volatility}

Consider the conditional mean of financial returns, as follows:

$$
y_{t}=E\left(y_{t} \mid I_{t-1}\right)+\varepsilon_{t},
$$

where the financial returns, $y_{t}=\Delta \log P_{t}$, represent the log-difference in financial commodity or agricultural prices, $P_{t}, I_{t-1}$ is the information set at time $t-1$, and $\varepsilon_{t}$ is a conditionally heteroskedastic error term, or returns shock. In order to derive conditional volatility specifications, it is necessary to specify the stochastic processes underlying the returns shocks, $\varepsilon_{t}$.

The most popular univariate conditional volatility model, GARCH model, is discussed below. Consider the random coefficient $\mathrm{AR}(1)$ process underlying the return shocks, $\varepsilon_{t}$ :

$$
\varepsilon_{t}=\phi_{t} \varepsilon_{t-1}+\eta_{t}
$$

where

$\phi_{t} \sim i i d(0, \alpha), \alpha \geq 0$,

$\eta_{t} \sim \operatorname{iid}(0, \omega), \omega \geq 0$,

$\eta_{t}=\varepsilon_{t} / \sqrt{h_{t}}$ is the standardized residual, with $h_{t}$ defined below.

Tsay (1987) derived the ARCH (1) model of Engle (1982) from equation (2) as:

$$
h_{t} \equiv E\left(\varepsilon_{t}^{2} \mid I_{t-1}\right)=\omega+\alpha \varepsilon_{t-1}^{2}
$$

where $h_{t}$ represents conditional volatility, and $I_{t-1}$ is the information set available at time $t-1$. A lagged dependent variable, $h_{t-1}$, is typically added to equation (3) to improve the sample fit:

$$
h_{t} \equiv E\left(\varepsilon_{t}^{2} \mid I_{t-1}\right)=\omega+\alpha \varepsilon_{t-1}^{2}+\beta h_{t-1} .
$$

From the specification of equation (2), it is clear that both $\omega$ and $\alpha$ should be positive as they are the unconditional variances of two different stochastic processes. Given the non-normality of the returns shocks, the Quasi-Maximum Likelihood Estimators (QMLE) of the parameters have been shown to be consistent and asymptotically normal in several papers.

\subsection{Multivariate Conditional Volatility}

The multivariate extension of the univariate ARCH and GARCH models is given in Baba et al. (1985) and Engle and Kroner (1995). In order to establish volatility spillovers in a multivariate framework, it is useful to define the multivariate extension of the relationship between the returns shocks and the standardized residuals, that is, $\eta_{t}=\varepsilon_{t} / \sqrt{h_{t}}$. The multivariate extension of equation (1), namely $y_{t}=E\left(y_{t} \mid I_{t-1}\right)+\varepsilon_{t}$, can remain 
unchanged by assuming that the three components are now $m \times 1$ vectors, where $m$ is the number of financial assets. The multivariate definition of the relationship between $\varepsilon_{t}$ and $\eta_{t}$ is given as:

$$
\varepsilon_{t}=D_{t}^{1 / 2} \eta_{t}
$$

where $D_{t}=\operatorname{diag}\left(h_{1 t}, h_{2 t}, \ldots, h_{m t}\right)$ is a diagonal matrix comprising the univariate conditional volatilities.

Define the conditional covariance matrix of $\varepsilon_{t}$ as $Q_{t}$. As the $m \times 1$ vector, $\eta_{t}$, is assumed to be iid for all $m$ elements, the conditional correlation matrix of $\varepsilon_{t}$, which is equivalent to the conditional correlation matrix of $\eta_{t}$, is given by $\Gamma_{t}$. Therefore, the conditional expectation of (5) is defined as:

$$
Q_{t}=D_{t}^{1 / 2} \Gamma_{t} D_{t}^{1 / 2}
$$

Equivalently, the conditional correlation matrix, $\Gamma_{t}$, can be defined as:

$$
\Gamma_{t}=D_{t}^{-1 / 2} Q_{t} D_{t}^{-1 / 2}
$$

Equation (6) is useful if a model of $\Gamma_{t}$ is available for purposes of estimating $Q_{t}$, whereas (7) is useful if a model of $Q_{t}$ is available for purposes of estimating $\Gamma_{t}$. Equation (6) is convenient for a discussion of volatility spillover effects, while both equations (6) and (7) are instructive for a discussion of asymptotic properties. As the elements of $D_{t}$ are consistent and asymptotically normal, the consistency of $Q_{t}$ in (6) depends on consistent estimation of $\Gamma_{t}$, whereas the consistency of $\Gamma_{t}$ in (7) depends on consistent estimation of $Q_{t}$. As both $Q_{t}$ and $\Gamma_{t}$ are products of matrices, with inverses in (7), neither the QMLE of $Q_{t}$ nor $\Gamma_{t}$ will be asymptotically normal based on the definitions given in equations (6) and (7).

\subsection{Diagonal BEKK}

The Diagonal BEKK model can be derived from a vector random coefficient autoregressive process of order one, which is the multivariate extension of the univariate process given in equation (2):

$$
\varepsilon_{t}=\Phi_{t} \varepsilon_{t-1}+\eta_{t},
$$

where

$\varepsilon_{t}$ and $\eta_{t}$ are $m \times 1$ vectors,

$\Phi_{t}$ is an $m \times m$ matrix of random coefficients,

$\Phi_{t} \sim \operatorname{iid}(0, A), A$ is positive definite,

$\eta_{t} \sim \operatorname{iid}(0, C), C$ is an $m \times m$ matrix.

Vectorization of a full matrix $A$ to vec $A$ can have dimension as high as $m^{2} \times m^{2}$, whereas vectorization of a symmetric matrix $A$ to vech $A$ can have a smaller dimension of $m(m+1) / 2 \times m(m+1) / 2$. In a case where $A$ is a diagonal matrix, with $a_{i i}>0$ for all $i=1, \ldots, m$ and $\left|b_{j j}\right|<1$ for all $j=1, \ldots, m$, so that $A$ has dimension $m \times m$, McAleer et al. (2008) showed that the multivariate extension of $\operatorname{GARCH}(1,1)$ from equation (8) is given as the Diagonal BEKK model, namely:

$$
Q_{t}=C C^{\prime}+A \varepsilon_{t-1} \varepsilon_{t-1}^{\prime} A^{\prime}+B Q_{t-1} B^{\prime},
$$

where $A$ and $B$ are both diagonal matrices, though the last term in equation (9) need not come from an underlying stochastic process. The diagonality of the positive definite matrix $A$ is essential for matrix multiplication as $\varepsilon_{t-1} \varepsilon_{t-1}^{\prime}$ is an $m \times m$ matrix; otherwise equation (9) could not be derived from the vector random coefficient autoregressive process in equation (8).

\subsection{Full, Triangular and Hadamard BEKK}

The full BEKK model in Baba et al. (1985) and Engle and Kroner (1995), who do not derive the model from an underlying stochastic process, is presented as:

$$
Q_{t}=C C^{\prime}+A \varepsilon_{t-1} \varepsilon_{t-1}^{\prime} A^{\prime}+B Q_{t-1} B^{\prime},
$$

except that $A$ and (possibly) $B$ in equation (10) are now both full matrices, rather than the diagonal matrices that were derived in equation (9) using the stochastic process in equation (8). The full BEKK model can be replaced by the triangular or Hadamard (element-by-element multiplication) BEKK models, with similar problems of identification and (lack of) existence.

A fundamental technical problem is that the full, triangular and Hadamard BEKK models cannot be derived from any known underlying stochastic processes, which means there are no regularity conditions (except by 
assumption) for checking the internal consistency of the alternative models, and consequently no valid asymptotic properties of the QMLE of the associated parameters (except by assumption).

Moreover, as the number of parameters in a full BEKK model can be as much as $3 m(m+1) / 2$, the "curse of dimensionality" will be likely to arise, which means that convergence of the estimation algorithm can become problematic and less reliable when there is a large number of parameters to be estimated.

\subsection{Granger Causality, Volatility Spillovers, and Optimal Hedge Ratios}

McAleer et al. (2008) showed that the QMLE of the parameters of the Diagonal BEKK model were consistent and asymptotically normal, so that standard statistical inference on testing hypotheses is valid. Moreover, as $Q_{t}$ in (9) can be estimated consistently, $\Gamma_{t}$ in equation (7) can also be estimated consistently.

The Diagonal BEKK model is given as equation (9), where the matrices $A$ and $B$ are given as:

$$
A=\left[\begin{array}{ccc}
a_{11} & \cdots & 0 \\
\vdots & \ddots & \vdots \\
0 & \cdots & a_{m m}
\end{array}\right], \quad B=\left[\begin{array}{ccc}
b_{11} & \cdots & 0 \\
\vdots & \ddots & \vdots \\
0 & \cdots & b_{m m}
\end{array}\right]
$$

The Diagonal BEKK model permits a test of Co-volatility Spillover effects, which is the effect of a shock in commodity $j$ at $t-1$ on the subsequent co-volatility between $j$ and another commodity at $t$. Given the Diagonal BEKK model, as expressed in equations (9) and (10), the subsequent co-volatility must only be between commodities $j$ and $i$ at time $t$.

Chang et al. (2015) define Full and Partial Volatility and Covolatility Spillovers in the context of Diagonal and Full BEKK models. Volatility spillovers are defined as the delayed effect of a returns shock in one asset on the subsequent volatility or covolatility in another asset. Therefore, a model relating $Q_{t}$ to returns shocks is essential, and this will be addressed in the following sub-section. Spillovers can be defined in terms of full volatility spillovers and full covolatility spillovers, as well as partial covolatility spillovers, as follows, for $i, j, k=1, \ldots, m$ :

(1) Full volatility spillovers: $\partial Q_{i t} / \partial \varepsilon_{k t-1}, k \neq i$;

(2) Full covolatility spillovers: $\partial Q_{i j t} / \partial \varepsilon_{k t-1}, i \neq j, k \neq i, j$;

(3) Partial covolatility spillovers: $\partial Q_{i j t} / \partial \varepsilon_{k t-1}, i \neq j, k=$ either $i$ or $j$.

Full volatility spillovers occur when the returns shock from financial asset $k$ affects the volatility of a different financial asset $i$.

Full covolatility spillovers occur when the returns shock from financial asset $k$ affects the covolatility between two different financial assets, $i$ and $j$.

Partial covolatility spillovers occur when the returns shock from financial asset $k$ affects the covolatility between two financial assets, $i$ and $j$, one of which can be asset $k$.

When $m=2$, only spillovers (1) and (3) are possible as full covolatility spillovers depend on the existence of a third financial asset.

This leads to the definition of a Co-volatility Spillover Effect as:

$$
\frac{\partial H_{i j, t}}{\partial \varepsilon_{j, t-1}}=a_{i i} \times a_{j j} \times \varepsilon_{i, t-1}, i \neq j .
$$

As $a_{i i}>0$ for all $i$, a test of the co-volatility spillover effect is given as a test of the null hypothesis:

$$
H_{0}: a_{i i} a_{j j}=0
$$

which is a test of the significance of the estimate of $a_{i i} a_{j j}$ in the following co-volatility spillover effect, as $\varepsilon_{i, t-1} \neq 0$ :

$$
\frac{\partial H_{i j, t}}{\partial \varepsilon_{j, t-1}}=a_{i i} a_{j j} \varepsilon_{i, t-1}, i \neq j
$$


If $H_{0}$ is rejected against the alternative hypothesis, $H_{1}: a_{i i} a_{j j} \neq 0$, there is a spillover from the returns shock of commodity $j$ at $t$ - 1 to the co-volatility between commodities $i$ and $j$ at $t$ that depends only on the returns shock of commodity $i$ at $t$-1. It should be emphasized that the returns shock of commodity $j$ at $t-1$ does not affect the co-volatility spillover of commodity $j$ on the co-volatility between commodities $i$ and $j$ at $t$. Moreover, spillovers can and do vary for each observation $t$ - 1 , so that the empirical results average co-volatility spillovers will be presented, based on the average return shocks over the sample period.

For the multivariate conditional mean returns equation:

$$
y_{i t}=E\left(y_{i t} \mid I_{t-1}\right)+\varepsilon_{i t}, i=1,2, \cdots, m,
$$

the bivariate random coefficient autoregressive process for $\varepsilon_{i t}$ is given as:

$$
\varepsilon_{i t}=\phi_{i t} \varepsilon_{i t-1}+\phi_{j t} \varepsilon_{j t-1}+\eta_{i t}, i \neq j,
$$

where

$\phi_{i t} \sim \operatorname{iid}\left(0, \alpha_{i}\right), \alpha_{i} \geq 0$,

$\phi_{j t} \sim \operatorname{iid}\left(0, \alpha_{j}\right), \alpha_{j} \geq 0$,

$\eta_{i t} \sim \operatorname{iid}\left(0, \omega_{i}\right), \omega_{i} \geq 0$,

$\eta_{i t}=\varepsilon_{i t} / \sqrt{h_{i t}}$ is the standardized residual,

$h_{i t}$ is the conditional volatility obtained by setting $\phi_{j t}=0$ in bivariate equation (16):

$$
\begin{gathered}
\varepsilon_{i t}=\phi_{i t} \varepsilon_{i t-1}+\eta_{i t}, \\
E\left(\varepsilon_{i t}^{2} \mid I_{t-1}\right) \equiv h_{i t}=\omega_{i}+\alpha_{i} \varepsilon_{i t-1}^{2} .
\end{gathered}
$$

Adding another commodity, as in the bivariate equation (16), gives:

$$
\begin{aligned}
& \varepsilon_{i t}=\phi_{i t} \varepsilon_{i t-1}+\phi_{j t} \varepsilon_{j t-1}+\eta_{i t}, i \neq j, \\
& E\left(\varepsilon_{i t}^{2} \mid I_{t-1}\right) \equiv h_{i t}=\omega_{i}+\alpha_{i} \varepsilon_{i t-1}^{2}+\alpha_{j} \varepsilon_{j t-1}^{2},
\end{aligned}
$$

while adding first-order lags of $h_{i t}$ and $h_{j t}$ gives:

$$
h_{i t}=\omega_{i}+\alpha_{i} \varepsilon_{i t-1}^{2}+\alpha_{j} \varepsilon_{j t-1}^{2}+\beta_{i} h_{i t-1}+\beta_{j} h_{j t-1},
$$

where

$$
\alpha_{i} \geq 0, \alpha_{j} \geq 0, \beta_{i} \in(-1,1), \beta_{j} \in(-1,1) .
$$

The null hypothesis of non-causality in volatility is given as a test of:

$$
H_{0}: \alpha_{j}=\beta_{j}=0 .
$$

Based on the empirical results, dynamic hedging strategies using optimal hedge ratios are suggested to analyse market fluctuations in the spot and futures returns and volatility of carbon emissions, crude oil and coal prices.

Using the hedge ratio: $R_{H, t}=R_{S, t}-\gamma_{t} R_{F, t}$ and its variance, namely:

$$
\operatorname{var}\left(R_{H, t} \mid \Omega_{t-1}\right)=\operatorname{var}\left(R_{S, t} \mid \Omega_{t-1}\right)-2 \gamma_{t} \operatorname{cov}\left(R_{S, t}, R_{F, t} \mid \Omega_{t-1}\right)+\gamma_{t}^{2} \operatorname{var}\left(R_{F, t} \mid \Omega_{t-1}\right),
$$

the optimal hedge ratio is given as:

$$
\gamma_{t} \mid \Omega_{t-1}=\operatorname{cov}\left(R_{S, t}, R_{F, t} \mid \Omega_{t-1}\right) / \operatorname{var}\left(R_{F, t} \mid \Omega_{t-1}\right) .
$$

\section{CONCLUDING REMARKS}

The paper discussed recent research that showed efforts to limit climate change have been focusing on the reduction of carbon dioxide emissions over other greenhouse gases or air pollutants. Many countries have paid great attention to carbon emissions in order to improve air quality and public health. The largest source of carbon emissions from human activities in many countries in Europe and around the world has been from burning fossil fuels. 
The prices of both fuel and carbon emissions can and do have simultaneous and contemporaneous effects on each other. Owing to the importance of carbon emissions and their interconnection to the prices, financial returns and associated volatilities of fossil fuels, and the possibility of Granger causality in spot and futures prices, returns and volatility of carbon emissions, it is not surprising that crude oil and coal, and their interactions with carbon emission prices, returns and volatility, have recently become very important for public policy and an associated research topic.

For the USA, daily spot and futures prices are available for crude oil and coal, but there are no daily spot or futures prices for carbon emissions. For the EU, there are no daily spot prices for coal or carbon emissions, but there are daily futures prices for crude oil, coal and carbon emissions. For this reason, daily prices were used to analyse Granger causality and volatility spillovers in spot and futures prices of carbon emissions, crude oil, and coal.

A quasi likelihood ratio (QLR) test was developed to test the multivariate conditional volatility Diagonal BEKK model, which has valid regularity conditions and asymptotic properties, against the alternative Full BEKK model, which has valid regularity conditions and asymptotic properties only under the null hypothesis of zero off-diagonal elements. In short, Full BEKK has no desirable mathematical or statistical properties, except either under the null hypothesis of zero off-diagonal elements of the weighting matrix, or simply by assumption. In the empirical analysis, DBEKK was rejected against the Full BEKK model for EU futures returns, but DBEKK was not rejected against Full BEKK for US spot returns. Therefore, further work would seem to be required for DBEKK in the case of EU futures returns, whereas DBEKK is empirically supported by the data for US spot returns.

Dynamic hedging strategies using optimal hedge ratios were suggested to analyse market fluctuations in the spot and futures returns and volatility of carbon emissions, crude oil and coal prices. It was suggested that the futures and spot prices of carbon emissions, coal and oil should be considered contemporaneously and simultaneously in a portfolio that links the prices, returns and volatilities of carbon emissions to the use of fossil fuels. It would also be sensible to use the prices in both markets to hedge carbon emission price returns in the EU against both coal and oil spot price returns in the USA.

\section{ACKNOWLEDGMENTS}

The first author wishes to thank the National Science Council, Ministry of Science and Technology (MOST), Taiwan, and the second author is grateful to the National Science Council, Ministry of Science and Technology (MOST), Taiwan and the Australian Research Council.

\section{REFERENCES}

Baba, Y., Engle, R.F., Kraft, D., and Kroner, K.F. (1985). Multivariate simultaneous generalized ARCH, Unpublished manuscript, Department of Economics, University of California, San Diego, CA, USA.

Chang, C.-L., Li, Y.-Y., and McAleer, M. (2015). Volatility Spillovers Between Energy and Agricultural Markets: A Critical Appraisal of Theory and Practice, Tinbergen Institute Discussion Paper 15-077/III, Tinbergen Institute.

Chang, C.-L. and McAleer, M. (2017). A simple test for causality in volatility, Econometrics, 5(1:15), 1-5.

Engle, R.F. (1982). Autoregressive conditional heteroskedasticity with estimates of the variance of United Kingdom inflation, Econometrica, 50(4), 987-1007.

Engle, R.F. and Kroner, K.F. (1995). Multivariate simultaneous generalized ARCH, Econometric Theory, 11(1), 122-150.

Granger, C.W.J. (1980). Testing for causality: A personal viewpoint, Journal of Economic Dynamics and Control, 2, 329-352.

Hafner, C.M. and Herwartz, H. (2006). A Lagrange multiplier test for causality in variance, Economics Letters, 93(1), 137-141.

McAleer, M., Chan, F., Hoti, S., and Lieberman, O. (2008). Generalized autoregressive conditional correlation, Econometric Theory, 24(6), 1554-1583.

Tsay, R.S. (1987). Conditional heteroscedastic time series models, Journal of the American Statistical Association, 82(398), 590-604. 